\title{
NUCLEOSYNTHESIS IN THE EARLY GALAXY
}

\author{
F. Montes, ${ }^{1,2,3}$ T. C. Beers, ${ }^{2,4}$ J. Cowan, ${ }^{5}$ T. Elliot,${ }^{2,3,6}$ K. Farouqi, ${ }^{7}$ R. Gallino, ${ }^{8}$ \\ M. Heil, ${ }^{1}$ K.-L. Kratz, ${ }^{7,9}$ B. Pfeiffer, ${ }^{7}$ M. Pignatari, ${ }^{8}$ and H. Schatz ${ }^{2,3,6}$ \\ Received 2007 February 23; accepted 2007 August 31
}

\begin{abstract}
Recent observations of $r$-process-enriched metal-poor star abundances reveal a nonuniform abundance pattern for elements $Z \leq 47$. Based on noncorrelation trends between elemental abundances as a function of Eu richness in a large sample of metal-poor stars, it is shown that the mixing of a consistent and robust light element primary process (LEPP) and the $r$-process pattern found in $r$-II metal-poor stars explains such apparent nonuniformity. Furthermore, we derive the abundance pattern of the LEPP from observation and show that it is consistent with a missing component in the solar abundances when using a recent $s$-process model. As the astrophysical site of the LEPP is not known, we explore the possibility of a neutron-capture process within a site-independent approach. It is suggested that scenarios with neutron densities $n_{n} \leq 10^{13} \mathrm{~cm}^{-3}$ or in the range $n_{n} \geq 10^{24} \mathrm{~cm}^{-3}$ best explain the observations.
\end{abstract}

Subject headings: Galaxy: abundances — nuclear reactions, nucleosynthesis, abundances — stars: formation

Online material: color figures

\section{INTRODUCTION}

The $r$-process is responsible for the origin of about half of the heavy isotopes beyond the iron group in nature, yet its site is still not determined with certainty (Cowan et al. 1991, 2006; Truran et al. 2002). The $r$-process involves extremely unstable nuclei, and in order for neutron captures to overcome the correspondingly short $\beta$-decay rates, typical conditions with neutron densities in excess of $10^{20} \mathrm{~cm}^{-3}$ and a process duration of less than $\approx 5 \mathrm{~s}$ are required (Kratz et al. 1993). $R$-process models have had difficulties in obtaining such conditions in realistic astrophysical environments. One promising scenario and one of the most studied is the neutrino-driven wind off a proto-neutron star in core-collapse supernovae (Woosley \& Hoffman 1992; Takahashi et al. 1994; Wanajo et al. 2001; Thompson et al. 2001; K. Farouqi et al. 2008, in preparation). Alternative scenarios include neutron star mergers (Freiburghaus et al. 1999; Rosswog et al. 1999; Goriely et al. 2005), jets in core-collapse supernovae (Cameron 2001), and gamma-ray bursts (Surman \& McLaughlin 2005). The $s$-process is responsible for creating roughly the other half of the isotopes beyond the iron group, while the $p$-process has significant contributions only to the relatively rare isotopes on the proton-rich side of the nuclei chart. It has also been recognized that to correctly reproduce the solar system $s$-process abundances, at least two different components are required (Käppeler et al. 1982): the

\footnotetext{
1 Gesellschaft für Schwerionenforschung, D-64220 Darmstadt, Germany.

2 Joint Institute for Nuclear Astrophysics, http://www.jinaweb.org.

3 National Superconducting Cyclotron Laboratory, Michigan State University, East Lansing, MI 48824.

4 Department of Physics and Astronomy and Center for the Study of Cosmic Evolution, Michigan State University, East Lansing, MI 48824.

5 Homer L. Dodge Department of Physics and Astronomy, University of Oklahoma, Norman, OK 73019.

${ }^{6}$ Department of Physics and Astronomy, Michigan State University, East Lansing, MI 48824.

${ }^{7}$ Virtuelles Institut für Struktur der Kerne und Nukleare Astrophysik, D-55128 Mainz, Germany.

${ }^{8}$ Dipartimento di Fisica Generale, Universitá di Torino, Via Pietro Giuria 1, I10125 Torino, Italy.

9 Max-Planck-Institut für Chemie, Otto-Hahn-Institut, Joh.-Joachim-BecherWeg 27, D-55128 Mainz, Germany.
}

weak $s$-process component responsible for creating $s$-isotopes with $A \leq 90$ (Raiteri et al. 1992; Pignatari et al. 2006) and the main $s$-process responsible for the heavier $s$-isotopes (Arlandini et al. 1999; Travaglio et al. 2004; Cristallo et al. 2006). A third $s$-process component, called "strong $s$-process," was envisaged by Clayton \& Rassbach (1967) at the termination of the $s$-process to account for essentially about $50 \%$ of solar ${ }^{208} \mathrm{~Pb}$. This component was recently recognized as the outcome of asymptotic giant branch (AGB) stars of low metallicity (Travaglio et al. 2001).

The solar system $r$-process abundances are often inferred by using the calculated $s$ - and $p$-abundances and subtracting them from the observed solar system abundances. If there is an additional nucleosynthesis process creating only small amounts of residual abundances, its contribution may be "hidden" in the sodefined solar system $r$-process abundances.

In addition to the solar system abundance distribution, observations of elemental abundances in unevolved metal-poor halo stars can provide important clues about nucleosynthesis events in the early Galaxy. These stars are old and preserve in their photospheres the abundance composition at the location and time of their formation. In particular, a subclass of extremely metal-poor $([\mathrm{Fe} / \mathrm{H}] \approx-3)$ but Eu-enhanced stars $([\mathrm{Eu} / \mathrm{Fe}]>0.5)$ exhibits what appears to be a pure $r$-process abundance pattern for the heavy $r$-process elements $Z \geq 56$ and $Z<83$. This pattern is remarkably stable from star to star and in excellent agreement with the contribution of the $r$-process to the solar abundances. A few of these stars have been found to date, with CS 22892-052 (Sneden et al. 2003) being the prime example. Since they are thought to exhibit the abundance pattern produced by a single or at most a few $r$-process events in the early Galaxy, the stability of the observed abundance pattern and the good agreement with the solar system $r$-process contribution imply that $r$-process events generate a universal abundance distribution. The universality of the abundance pattern of the heavy $r$-process elements seems not to extend to the actinides Th and $\mathrm{U}$, where some star-to-star scatter has been found in some cases (Hill et al. 2002; Goriely \& Arnould 2001; Schatz et al. 2002; Honda et al. 2004).

While the $r$-process abundance pattern for $56 \leq Z<83$ is stable from star to star, the overall level of enrichment with respect to iron (e.g., $[\mathrm{Eu} / \mathrm{Fe}])$ shows a very large star-to-star scatter. This 
implies that very metal-poor halo stars sample a largely unmixed early Galaxy and that the $r$-process occurs in at most $2 \%-10 \%$ of iron-producing core-collapse supernovae (Truran et al. 2002; assuming core-collapse supernovae are the site of the $r$-process).

However, this simple picture breaks down for the lighter neutroncapture elements with $Z \leq 47$. The abundances of these elements measured in the strongly Eu-enhanced stars once normalized to Eu do not agree entirely with the solar system $r$-process residual pattern. In particular, the abundances of $\mathrm{Y}, \mathrm{Mo}, \mathrm{Rh}, \mathrm{Pd}$, and $\mathrm{Ag}$ are consistently below the solar system values. This indicates that either the $r$-process in this region is not robust and depends on the astrophysical condition or metallicity, or that the $r$-process observed in these stars is not the only nucleosynthesis process leading to the abundance pattern obtained by subtracting the $s$ - and $p$-processes from the solar abundances (Pfeiffer et al. 2001a, $2001 \mathrm{~b}$ ). There is additional evidence for such a second process being present in the early Galaxy; Wasserburg et al. (1996) and Qian \& Wasserburg (2000) first proposed the existence of two different $r$-process sites or components based on meteoritic evidence of live light $r$-process ${ }^{129} \mathrm{I}\left(T_{1 / 2}=15.7 \mathrm{Myr}\right)$ in the early solar system compared to the heavy $r$-process isotopes such as ${ }^{182} \mathrm{Hf}\left(T_{1 / 2}=8.9 \mathrm{Myr}\right.$; Vockenhuber et al. 2004). Wasserburg \& Qian (2000a, 2000b) and Qian \& Wasserburg $(2001,2003)$ also proposed that two $r$-processes together with a "prompt" nucleosynthesis contribution could explain the metal-poor star abundance observations available at the time. However, it has been pointed out that the proposal of different $r$-process sites for the isotopes in the second $r$-process peak from I to Te (including ${ }^{129} \mathrm{I}$ ) and for isotopes of $\mathrm{Ba}$ and beyond (including ${ }^{182} \mathrm{Hf}$ ) are difficult to reconcile with $r$-process models and the known nuclear physics at the $N=82$ shell gap (Kratz et al. 2007). McWilliam (1998), Burris et al. (2000), Norris et al. (2001), Johnson \& Bolte (2002), Lambert \& Allende Prieto (2002), Truran et al. (2002), Honda et al. (2004), and Barklem et al. (2005) reported the observation of a large scatter in $[\mathrm{Sr} / \mathrm{Ba}]$ in low-metallicity stars. This has been interpreted as further evidence for a second, independent process that produces Sr but little or no Ba at low metallicities. Travaglio et al. (2004) demonstrated that the same is true for $\mathrm{Y}$ and $\mathrm{Zr}$, and they postulated a light element primary process (LEPP) producing $\mathrm{Sr}$, Y, and Zr but little Eu and Ba. Truran et al. (2002) pointed out that there is in fact a noncorrelation of $[\mathrm{Sr} / \mathrm{Ba}]$ and $[\mathrm{Ba} / \mathrm{Fe}]$ in some stars showing very large $[\mathrm{Sr} / \mathrm{Ba}]$ ratios but little $\mathrm{Ba}$. Again, this can be explained by the presence of a second process producing mainly Sr that happens to dominate the composition in such stars. A similar noncorrelation was found by Otsuki et al. (2006) for a few stars in the globular cluster M15. Aoki et al. (2005) came to a similar conclusion based on trends in the behavior of $\mathrm{Y}$ and $\mathrm{Zr}$ as a function of Eu.

We show in $\S 2$ that such a noncorrelation can be found in all metal-poor, $r$-process-enriched stars and for additional light elements beyond $\mathrm{Sr}, \mathrm{Y}$, and $\mathrm{Zr}$. This includes previously noted "anomalies" such as the observed abundances in HD 122563 (Honda et al. 2006) that cannot be fit by an $r$ - or an $s$-process alone. Also included is the abundance pattern in the moderately $r$-element-enhanced star HD 221170 (Ivans et al. 2006), which does not exhibit the underabundance of light $r$-process elements with respect to the solar $r$-process contribution. We show that these "anomalies" are in fact part of a general trend that is consistent with a mixture of two processes in metal-poor stars, an $r$-process and a LEPP process exhibiting rather stable abundance patterns, which are mixed in varying proportions. In $\S 3$ we analyze the features of the newly derived LEPP abundance pattern in terms of a neutron-capture-process model to determine the astro- physical conditions required for this new process. Conclusions are presented in $\S 4$.

\section{ABUNDANCE CLUES}

The observed elemental abundances of metal-poor $([\mathrm{Fe} / \mathrm{H}]<$ $-1)$ and $r$-process-enriched $([\mathrm{Ba} / \mathrm{Eu}]<0)$ stars are shown in Figure 1 (Burris et al. 2000; Honda et al. 2004, 2006; Christlieb et al. 2004; Barklem et al. 2005). All abundances are normalized to $\mathrm{Eu}$, which is predominantly an $r$-process element $(\approx 95 \%$ of the total solar abundance). The heavy $r$-process elements with $Z \geq 56$ exhibit, within the observational errors, a constant ratio with respect to $\mathrm{Eu}$, independent of the Eu enrichment of the star. This ratio is consistent with the element ratio of the $r$-process contribution to the solar system. This indicates that the heavy elements are produced by the $r$-process that produces a universal abundance pattern with fixed element ratios consistent with the solar $r$-process abundance pattern. This is the same conclusion that has been drawn from the abundance pattern of the few very metal-poor, strongly $r$-process-enhanced stars where a large range of elemental abundance has been determined (see, e.g., the reviews by Truran et al. 2002; Cowan et al. 2006).

It is also obvious from Figure 1 that the lighter $r$-process elements behave very differently. Clearly, the $[\mathrm{Y} / \mathrm{Eu}],[\mathrm{Sr} / \mathrm{Eu}]$, and $[\mathrm{Zr} / \mathrm{Eu}]$ ratios are not constant but show a noncorrelation with the Eu enrichment. This noncorrelation indicates that a different process (the LEPP), which does not create substantial amounts of heavier $r$-process elements such as Eu, has contributed significantly to the abundances of Sr, Y, and Zr. Otsuki et al. (2006), studying six giants in the globular cluster M15, observed a similar relation for $\mathrm{Y}$ and $\mathrm{Zr}$ with respect to $\mathrm{Eu}$ and came to the same conclusion, but with very low statistics. We confirm their result with a much larger sample and show that this is true for all $r$-processenhanced, metal-poor stars. While observations require this process to be different in the sense that it does not always operate simultaneously with the process that produces the $r$-process abundances observed in highly $r$-process-enhanced stars $(r$-II, with $[\mathrm{Eu} / \mathrm{Fe}]>$ 1.0 and $[\mathrm{Ba} / \mathrm{Eu}]<0.0$, according to Beers \& Christlieb 2005), it is still possible that both processes occur in the same astrophysical object.

The $\left[\mathrm{X}_{i} / \mathrm{Eu}\right]$ versus $[\mathrm{Eu} / \mathrm{Fe}]$ slopes for the light $r$-process elements in Figure 1 are roughly consistent with -1 for $[\mathrm{Eu} / \mathrm{Fe}]$ that are not too large. This is a consequence of the correlation of the light $r$-process elements with $\mathrm{Fe}$ instead of Eu. As Travaglio et al. (2004) have shown for $\mathrm{Sr}, \mathrm{Y}$, and $\mathrm{Zr},\left[\mathrm{X}_{i} / \mathrm{Fe}\right]$ is roughly constant and shows a rather small scatter as a function of metallicity $[\mathrm{Fe} / \mathrm{H}]$. Because of $\left[\mathrm{X}_{i} / \mathrm{Eu}\right]=\left[\mathrm{X}_{i} / \mathrm{Fe}\right]-[\mathrm{Eu} / \mathrm{Fe}]$, this results in a -1 slope in $\left[\mathrm{X}_{i} / \mathrm{Eu}\right]$ versus $[\mathrm{Eu} / \mathrm{Fe}]$.

An interesting question is the behavior of other light $r$-process elements below $\mathrm{Ba}$. $\mathrm{Ag}$ would be a good indicator for the $r$-process, as $\approx 80 \%-86 \%$ (Arlandini et al. 1999; Burris et al. 2000; Travaglio et al. 2004) ${ }^{10}$ of the total solar abundance is produced in the $r$-process. Unfortunately, only metal-poor stars CS 22892-052 (Sneden et al. 2003), HD 155444 (Westin et al. 2000; C. Sneden 2006, private communication), BD +173248 (Cowan et al. 2002), CS 31082-001 (Hill et al. 2002), HD 221170, and $\mathrm{HD} 122563$ have published abundance yields of $\mathrm{Nb}, \mathrm{Mo}, \mathrm{Ru}, \mathrm{Rh}$, $\mathrm{Pd}$, and Ag. ${ }^{11}$ Nevertheless, although the statistics are low for Pd

\footnotetext{
10 A reanalysis of the calculations in Travaglio et al. (2004) indicates some modification in the values for the Ag entries in their Table 5. The corrected $s$-fraction is $14 \%$, and the $r$-residual is then $86 \%$.

11 Recent high-resolution spectroscopy of the extremely metal-poor star HD 88609 was recently reported in Honda et al. (2007) after the manuscript was completed, and it is not included in the discussion. Its elemental distribution is very similar to that observed in HD 122563.
} 

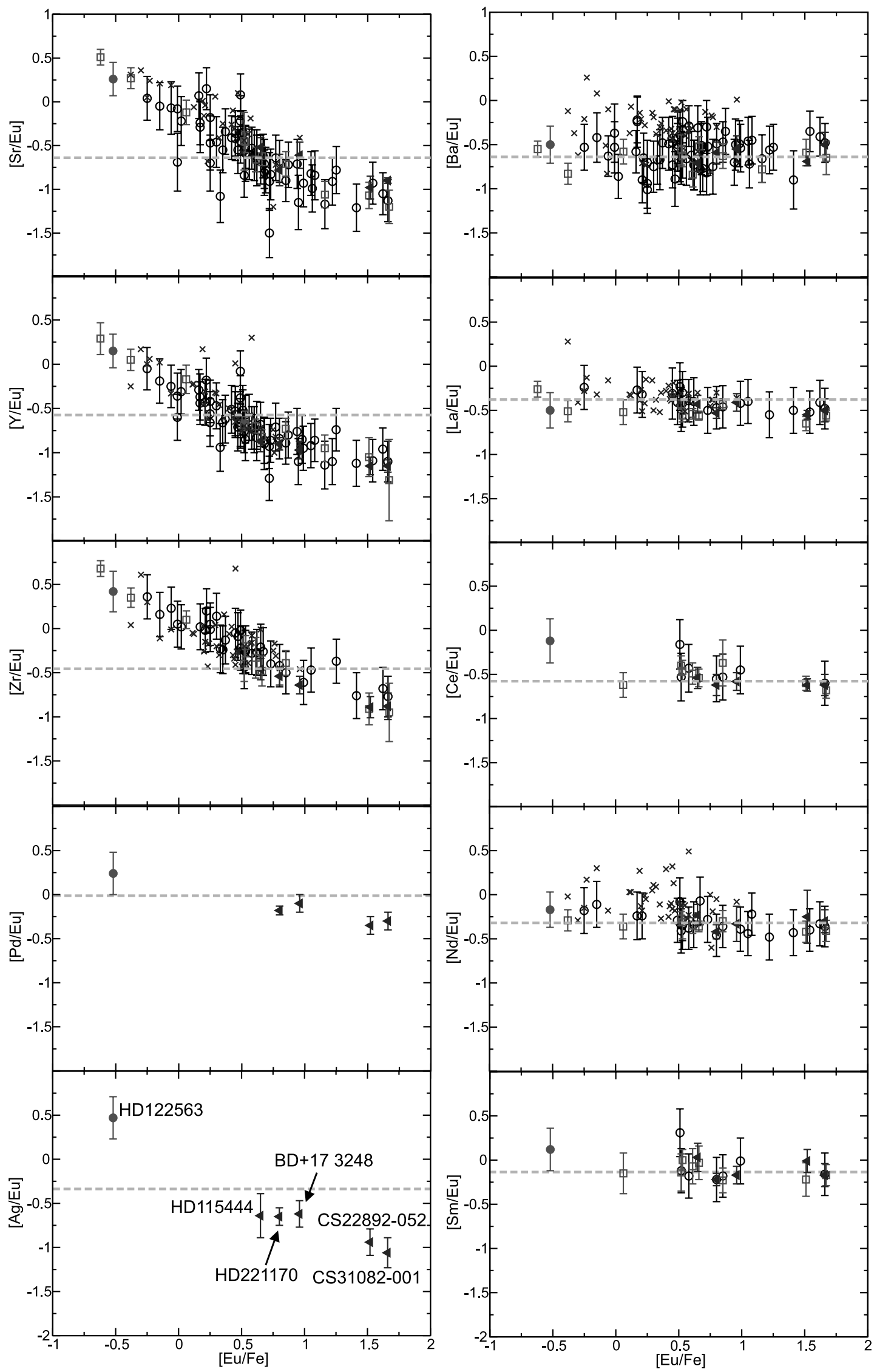

FIg. 1.-Abundance ratio of metal-poor stars as a function of $[\mathrm{Eu} / \mathrm{Fe}]$. Only stars with $[\mathrm{Ba} / \mathrm{Eu}]<0$ (except $\mathrm{HD} 122563$ ) and $[\mathrm{Fe} / \mathrm{H}]<-1$ are shown. Abundances represented by crosses were taken from Burris et al. (2000), squares are from Honda et al. (2004), open circles are from Christlieb et al. (2004) and Barklem et al. (2005), and filled circles are from Honda et al. (2006). Dashed lines are the respective $r$-process ratios. [See the electronic edition of the Journal for a color version of this figure.] 
and $\mathrm{Ag}$, the elemental abundances shown in Figure 1 are more consistent with an Eu noncorrelation as observed for $\mathrm{Sr}, \mathrm{Y}$, and $\mathrm{Zr}$ than with the constant ratio exhibited by the heavier $r$-process elements. We must caution, however, that the observed Ag abundances in these metal-poor stars may have some uncertainties. The atomic data for this element are well established, and the solar system abundance also appears well determined, but it is not clear whether non-LTE effects could have affected the abundance analysis, since the Ag abundances are based on (low-lying) neutral transitions as opposed to ion transitions.

Within their very low statistics (two to four data points), the abundances of $\mathrm{Nb}, \mathrm{Mo}$, and $\mathrm{Rh}$ are also consistent with the trend exhibited by $\mathrm{Sr}, \mathrm{Y}, \mathrm{Zr}, \mathrm{Pd}$, and $\mathrm{Ag}$. A possible exception is $\mathrm{Ru}$, which shows a flat trend, but it is not possible to make definite conclusions based on just three data points. Ru also has less established atomic data for the lines analyzed. We therefore conclude that the LEPP not only produces $\mathrm{Sr}, \mathrm{Y}$, and $\mathrm{Zr}$, but most likely all light elements between $\mathrm{Sr}$ and $\mathrm{Ag}$ observed in very low metallicity stars.

Figure 1 also shows the underproduction of $\mathrm{Sr}, \mathrm{Y}, \mathrm{Zr}, \mathrm{Ag}$, and $\mathrm{Pd}$ versus $\mathrm{Eu}$ with respect to the solar pattern for the most Eu-enriched stars. Clearly, this underproduction is a function of $\mathrm{Eu}$ enrichment. Ivans et al. (2006) recently pointed out that in HD $221170([\mathrm{Eu} / \mathrm{Fe}]=0.8)$, not only the heavy $r$-process elements but also the light $r$-process elements are in reasonable agreement with the solar $r$-process abundance pattern and do not show the pronounced underproduction of some elements, such as Ag and $\mathrm{Pd}$, as seen in other $r$-process-enhanced stars. Given the slopes indicated by the data displayed in Figure 1, one does indeed ex$\operatorname{pect}[\mathrm{Sr} / \mathrm{Eu}],[\mathrm{Y} / \mathrm{Eu}],[\mathrm{Zr} / \mathrm{Eu}],[\mathrm{Pd} / \mathrm{Eu}]$, and $[\mathrm{Ag} / \mathrm{Eu}]$ ratios close to the solar $r$-process value for moderately $r$-process-enriched stars around $[\mathrm{Eu} / \mathrm{Fe}]=0.8$.

Recently, Honda et al. (2006) reported seven new elemental abundances in the metal-poor star HD 122563 and observed an excess of light neutron-capture elements. This star has ratios of $[\mathrm{Ba} / \mathrm{Eu}]=-0.5,[\mathrm{Fe} / \mathrm{H}]=-2.7$, and $[\mathrm{Eu} / \mathrm{Fe}]=-0.5$, and the enhancement of light neutron-capture elements makes it a candidate for a LEPP-enhanced metal-poor star. The abundances of HD 122563 are also shown in Figure 1. They follow nicely the abundance trends found in all the other metal-poor stars, and this consistency makes us believe that HD 122563 has, in fact, a significant LEPP contribution.

There are some indications that the observed stable abundance pattern of the main $r$-process (except for $U$ and $T h$ ) extends to the light $r$-process elements. Figure 1 shows a flattening of the $\left[\mathrm{X}_{i} / \mathrm{Eu}\right]$ versus $[\mathrm{Eu} / \mathrm{Fe}]$ slopes for light $r$-process elements in the most enriched stars with $[\mathrm{Eu} / \mathrm{Fe}]>1.3$, where the main $r$-process component dominates. This is most clearly seen for $\mathrm{Sr}$ and $\mathrm{Y}$, but $\mathrm{Zr}, \mathrm{Pd}$, and $\mathrm{Ag}$ are not inconsistent with such a trend. In addition, within the error bars the $\left[\mathrm{X}_{i} / \mathrm{Eu}\right]$ scatter in the $[\mathrm{Eu} / \mathrm{Fe}]>1.3$ region is small and comparable to the heavier $r$-process elements. We therefore take the most Eu-enriched stars such as CS 22892052 and $\mathrm{CS} 31082-001([\mathrm{Eu} / \mathrm{Fe}]>+1.0)$ as representatives of a stable, universal $r$-process component (except for $\mathrm{U}$ and $\mathrm{Th}$ ). The picture that then emerges is that in less Eu-enriched stars an additional contribution from the LEPP to the light elements from $\mathrm{Sr}$ to Ag becomes visible. We show below that one then also obtains a LEPP abundance pattern that is fairly consistent from star to star, which again is a hint that our assumptions are justified.

One might argue that there is the possibility of a metallicity dependence of the $r$-process abundance pattern for the light $r$-process elements. Figure 2 shows the ratio $[\mathrm{Sr} / \mathrm{Eu}]$ as a function of metallicity. There is no indication of a metallicity dependence from these data, and at least for $[\mathrm{Fe} / \mathrm{H}]<-1$, they are consistent with a

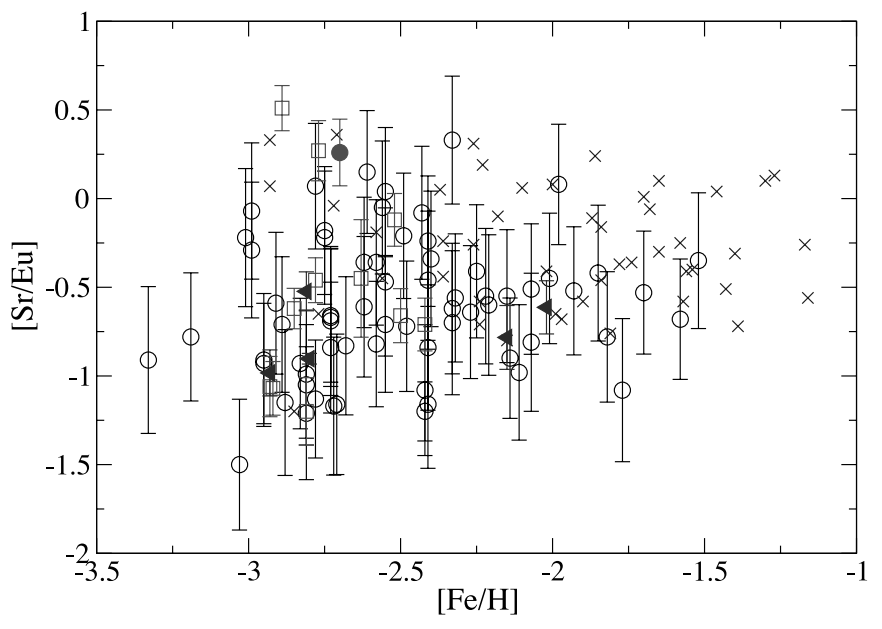

FIG. 2.-Same as Fig. 1, but for abundance ratio [Sr/Eu] of metal-poor stars as a function of metallicity [ $\mathrm{Fe} / \mathrm{H}]$. [See the electronic edition of the Journal for a color version of this figure.]

large scatter resulting from mixing light- and heavy-element nucleosynthesis processes at low metallicities and a gradual homogenization of the composition of the Galaxy as a function of metallicity.

In order to find the LEPP pattern in all the metal-poor stars studied, we use CS 31082-001 as an $r$-process-only star and determine the LEPP abundances in other stars by subtracting its abundance pattern normalized to Eu. This assumes that all Eu is made in the $r$-process. The resulting residual abundances shown in Figure 3 were scaled to $\mathrm{Zr}$ so that the patterns can be compared. As Figure 3 shows, the residual abundances are very consistent for all elements and for all stars shown. A similar result is obtained by using CS 22892-052 as a representative of an $r$-process-only star. The scatter of the data in Figure 3 measures variations within the light-element pattern. Clearly, the scatter is greatly reduced compared to $[\mathrm{X} / \mathrm{Fe}]$, which has already been shown for $\mathrm{Y}, \mathrm{Sr}$, and $\mathrm{Zr}$ (Travaglio et al. 2004). Here we show that the few data on Pd and $\mathrm{Ag}$ are consistent with similar behavior. Even though the error bars are large for high $[\mathrm{Eu} / \mathrm{Fe}]$, they become smaller for stars with significant LEPP contribution. The distribution of the data is consistent with no scatter, indicating a consistent pattern from star to star for the lighter elements.

We therefore conclude that the LEPP creates a uniform and unique pattern and that with a mixing of a robust $r$-process, the abundance composition of the other metal-poor stars can be obtained. For elements from $\mathrm{Sr}$ to $\mathrm{Ag}$, all weakly Eu-enriched stars show an overabundance with respect to CS 31082-001, simply reflecting the extra LEPP component. For elements heavier than $\mathrm{Ag}$, the LEPP enrichment is less significant, and for almost all of the stars only an upper limit in the abundance can be obtained.

To obtain information on the elements that are only weakly produced in the LEPP, one needs to look at the stars with the lowest $[\mathrm{Eu} / \mathrm{Fe}]$ where the LEPP most prominently dominates the composition. We believe that HD 122563 is an example of such a star. Therefore, having argued for the uniformity and uniqueness of the LEPP abundance pattern based on a number of stars, we now use HD 122563 to obtain our best estimate of the LEPP abundance pattern. We take the average of the known $r$-II stars and subtract this best estimate of the $r$-process from the HD 122563 abundance pattern assuming that $\mathrm{Eu}, \mathrm{Gd}, \mathrm{Dy}, \mathrm{Er}$, and $\mathrm{Yb}$ were solely produced in the $r$-process (i.e., scaling the main component to those elemental abundances). Both patterns are shown in Figure 4. The result is referred to as the "stellar LEPP" abundance 


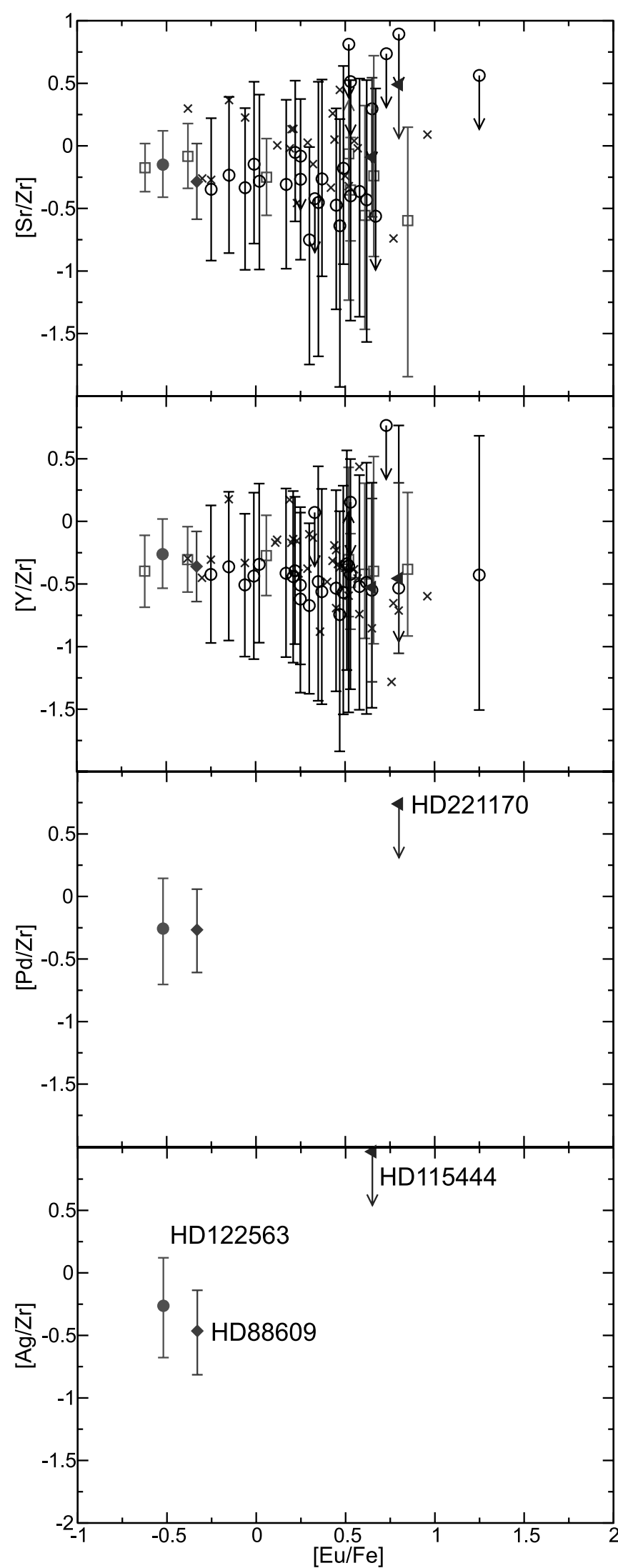

FIG. 3.- Same as Fig. 1, but for abundance obtained after subtracting a Eu-scaled CS 31082-001 abundance (main $r$-process) from metal-poor stars as a function of $[\mathrm{Eu} / \mathrm{Fe}]$. Diamonds represent abundances taken from Qian \& Wasserburg (2007). [See the electronic edition of the Journal for a color version of this figure.]

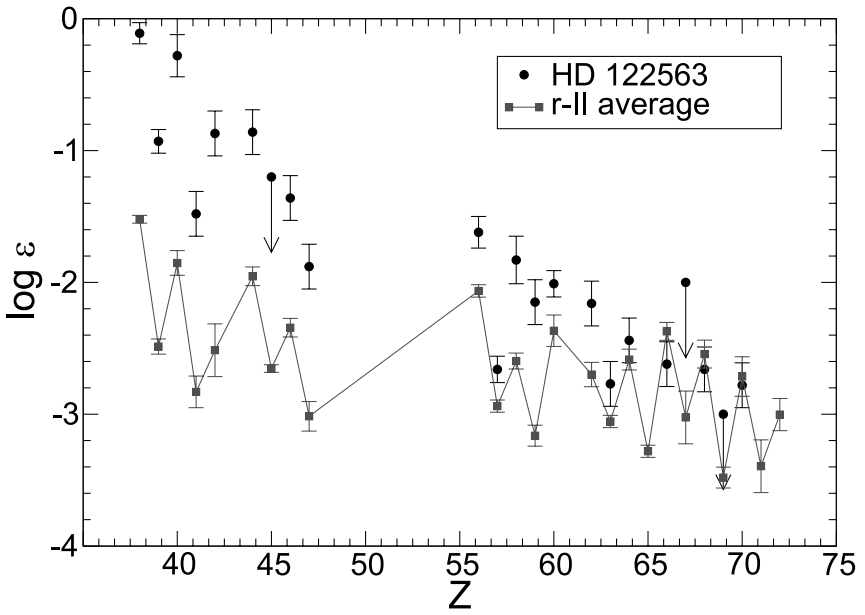

FIG. 4.-Abundance pattern of HD 122563 and scaled abundance pattern obtained by averaging $r$-II stars CS $31082-001$ and CS 22892-052. The average $r$-II star pattern was normalized to the Eu, Gd, Dy, Er, and Yb HD 122563 abundance. [See the electronic edition of the Journal for a color version of this figure.]

pattern in this paper, and it is shown in Figure 5. It is noteworthy that we find that some smaller amounts of $\mathrm{Ba}, \mathrm{Ce}, \mathrm{Pr}, \mathrm{Nd}, \mathrm{Sm}$, and $\mathrm{Eu}$ are still produced by the LEPP.

It would be desirable to obtain a more complete abundance pattern of more Eu-deficient metal-poor stars that exhibit the same (non)correlations as HD 122563. Candidates for such stars are $\mathrm{HD} 88609([\mathrm{Fe} / \mathrm{H}]=-2.93,[\mathrm{Eu} / \mathrm{Fe}]=-0.3)$, HD $13979([\mathrm{Fe} / \mathrm{H}]=-2.26,[\mathrm{Eu} / \mathrm{Fe}]=-0.4)$, and $\mathrm{HD} 4306$ $([\mathrm{Fe} / \mathrm{H}]=-2.7,[\mathrm{Eu} / \mathrm{Fe}]=-0.6)$. However, the abundances of elements from $\mathrm{Ru}$ to $\mathrm{Ag}$ have not yet been observed in these stars.

To determine the LEPP contribution to the solar system abundances, we subtracted the average of the known highly $r$-processenhanced stars from the abundance pattern obtained by subtracting the $s$ - and $p$-processes from the solar abundance. Any determination of the solar $r$-process abundances suffers from the uncertainties in predicting the $s$ - and $p$-process contributions. In particular, uncertainties in the neutron-capture cross sections and in the solar system abundances (Arlandini et al. 1999; Travaglio et al.

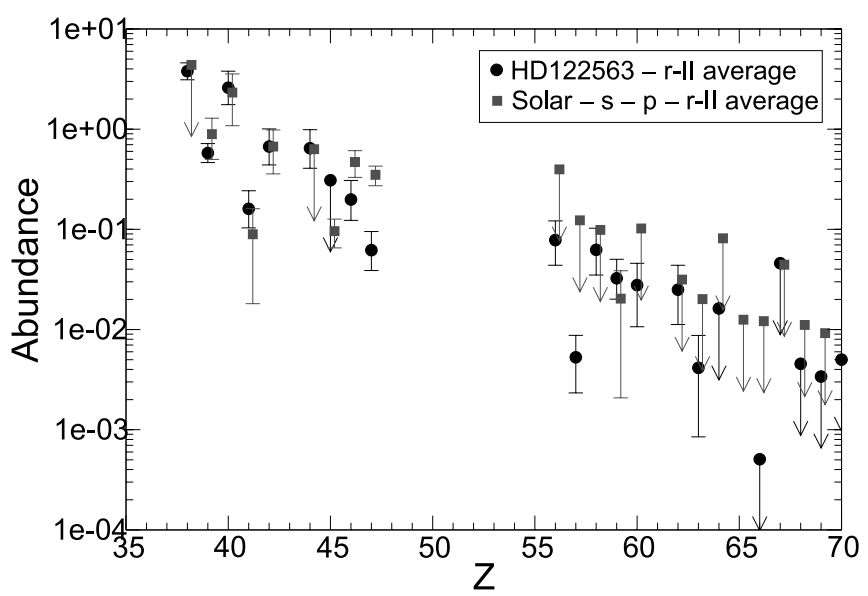

FIG. 5.-Abundance pattern created by the LEPP. The pattern represented by squares was created by subtracting the scaled average of CS 31082-001 and CS $22892-052$, the $s$ - and $p$-process contributions from the solar abundance (solar LEPP). The pattern represented by circles was obtained by subtracting the scaled average of CS 31082-001 and CS 22892-052 from HD 122563 (stellar LEPP) and scaling it to the solar LEPP Mo abundance. See text for explanation. [See the electronic edition of the Journal for a color version of this figure.] 
TABLE 1

LEPP Contribution to the Solar System Total Abundance

\begin{tabular}{|c|c|}
\hline Element & Percent \\
\hline $\mathrm{Sr}$ & $\leq 19$ \\
\hline Y & $19(8)$ \\
\hline $\mathrm{Zr}$ & $20(11)$ \\
\hline $\mathrm{Nb}$ & $13(10)$ \\
\hline Мo .......................... & $26(12)$ \\
\hline $\mathrm{Ru}$ & $\leq 34$ \\
\hline $\mathrm{Rh}$ & $28(9)$ \\
\hline Pd ....................... & $34(10)$ \\
\hline $\mathrm{Ag}$ & $72(16)$ \\
\hline $\mathrm{Ba}$ & $\leq 9$ \\
\hline La ....................... & $\leq 28$ \\
\hline $\mathrm{Ce}$ & $\leq 8$ \\
\hline $\operatorname{Pr}$ & $12(11)$ \\
\hline $\mathrm{Nd}$ & $\leq 12$ \\
\hline $\mathrm{Sm}$ & $\leq 12$ \\
\hline $\mathrm{Eu}$ & $\leq 21$ \\
\hline 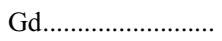 & $\leq 25$ \\
\hline
\end{tabular}

Note.-Solar LEPP as defined in the text.

2004) create uncertainties in the predicted $s$-process abundances which were taken into account in the calculations. The solar system abundances were taken from Anders \& Grevesse (1989) and Lodders (2003). The weak component of the $s$-process was included using the results of Raiteri et al. (1992). Different models have been used in the past for the main $s$-process component, which is the most important one for our study. Arlandini et al. (1999) used the average $s$-process yield from two AGB stellar models for a 1.5 and a $3 M_{\odot}$ star, both at metallicity $1 / 2 Z_{\odot}$. On the other hand, Travaglio et al. (2004) followed a Galactic chemical evolution model that used a range of masses and metallicities and also included intermediate-mass star $s$-process yields. For elements with $Z \geq 50$, both models agree within the error bars, and no major discrepancies are found. While for elements with $Z \leq 37$, Travaglio et al. (2004) produce relatively more $s$-material than Arlandini et al. (1999) due to the additional contribution in this region by AGB stars of intermediate mass (4-8 $\left.M_{\odot}\right)$, the opposite happens for elements in the range $38 \leq Z \leq 51$. The main differences in the resulting solar $r$-process contributions are therefore found for $\mathrm{Sr}, \mathrm{Y}$, and $\mathrm{Zr}$. Using the $s$-process contribution from Arlandini et al. (1999), the solar residuals exhibit smaller amounts of $\mathrm{Sr}, \mathrm{Y}$, and $\mathrm{Zr}$ material than when using the $s$-process calculations from Travaglio et al. (2004). Because the Travaglio et al. (2004) Galactic chemical evolution model is more complete and includes more relevant physics, it is the model that was used in our study. The effect of the $p$-process on the elemental abundance was included by assuming that it solely adds to abundances of proton-rich isotopes. Only the abundance of Mo and $\mathrm{Ru}$ are significantly modified by the $p$-process. The elemental LEPP contribution to the solar system abundances, which we now call the "solar LEPP" pattern, obtained by subtracting the $s-, p$-, and $r$-process (average of $r$-II stars) from the solar abundances, is shown in Table 1. Upper limits of the isotopic solar LEPP abundances were also obtained. Note that for $s$-only isotopes the LEPP abundances can be unambiguously calculated, since they do not have an $r$-process contribution.

Figure 5 compares the solar LEPP pattern with the stellar LEPP abundance pattern. We find rather good agreement for elements $\mathrm{Y}$, $\mathrm{Sr}, \mathrm{Zr}, \mathrm{Nb}, \mathrm{Mo}, \mathrm{Ru}$, and $\mathrm{Rh}$. We therefore propose that the LEPP observed in the abundances of metal-poor stars and the process that is responsible for filling in the residual obtained when subtracting from the solar abundances the $s$-process from Travaglio et al. (2004), the $r$-process component observed in the most Eu-enriched metal-poor stars, and the $p$-process are the same. The relative contributions of the LEPP to the solar system abundances are also in agreement with Ishimaru et al. (2005), who found indications that the LEPP (or weak $r$-process in their notation) contribution decreases with atomic number. The element Pd would be somewhat intermediate between $\mathrm{Sr}$, which is dominated by the LEPP, and $\mathrm{Ba}$, which is dominated by the main $r$-process. However, there are also some discrepancies between the stellar and solar LEPP patterns. The solar LEPP abundance of Pd is about a factor of 2 smaller than the stellar one, but still within $2 \sigma$ of the error bars. However, the solar LEPP abundance of Ag is 5 times less than the stellar LEPP abundance. Since Ag is mainly an $r$-process element $(\approx 80 \%-86 \%)$, it is unlikely that the $s$-process contribution is underestimated by more than a factor of 3 to account for the difference. As mentioned earlier, one possible explanation is the uncertainty of non-LTE effects in the abundance analysis of the metal-poor stars.

\section{ASTROPHYSICAL CONDITIONS}

The second nucleosynthesis process producing the lighter $r$-process elements postulated in the pioneering work of Wasserburg et al. (1996) has usually been assumed to be an $r$-process due to its required production of ${ }^{129} \mathrm{I}$. Consequently, Qian et al. (1998) did attempt to model its abundance pattern with a schematic strongly simplified $r$-process model based on the few observational data that were available at the time.

Traditionally, light $s$-process products have not been thought to be produced at very low metallicities. In particular, the weak $s$-process abundance contribution is negligible in extremely metalpoor stars with $[\mathrm{Fe} / \mathrm{H}] \approx-3$ to account for the abundance of $\mathrm{Sr}$, $\mathrm{Y}$, and $\mathrm{Zr}$, since the main neutron source in massive stars, ${ }^{22} \mathrm{Ne}$, is of secondary nature. Indeed, production of ${ }^{22} \mathrm{Ne}$ derives from the original CNO nuclei, first converted essentially to ${ }^{14} \mathrm{~N}$ during core $\mathrm{H}$-burning, then converted to ${ }^{18} \mathrm{O}$ by $\alpha$-capture at the beginning of core He-burning and further processed by $\alpha$-capture to ${ }^{22} \mathrm{Ne}$. Neutrons are released by the ${ }^{22} \mathrm{Ne}(\alpha, n)^{25} \mathrm{Mg}$ channel near core $\mathrm{He}$ exhaustion or during the following convective shell ${ }^{12} \mathrm{C}$ burning.

Furthermore, the main $s$-process occurs in low- and intermediatemass stars which have relatively long life spans and thus cannot explain the observation of metal-poor star abundances. The isotopic deficiencies of the Galactic chemical evolution $s$-process model of Travaglio et al. (2004) in the $s$-only isotopes ${ }^{86} \mathrm{Sr},{ }^{96} \mathrm{Mo}$, ${ }^{100} \mathrm{Ru},{ }^{104} \mathrm{Pd},{ }^{110} \mathrm{Cd},{ }^{116} \mathrm{Sn},{ }^{122-124} \mathrm{Te}$, and ${ }^{128,130} \mathrm{Xe}$, which are only produced with abundances of $70 \%-80 \%$ of the solar value, are problematic, since those isotopes should come entirely from the main $s$-process. Either a third $s$-process component has to be included to account for such deficiencies or there is a problem in their model. For this reason, the possible existence of a primary $s$-process contributing to abundances in this region cannot be excluded. In addition, Fröhlich et al. (2006) and Wanajo (2006) have recently suggested that the $\nu p$-process might contribute to the $\mathrm{Y}, \mathrm{Sr}$, and $\mathrm{Zr}$ abundances observed in metal-poor stars. The $\nu p$-process occurs in a proton-rich, neutrino-driven wind off a proto-neutron star and is therefore a primary process that could operate in the early Galaxy. While this process produces primarily neutron-deficient isotopes, it cannot be excluded observationally, as only elemental abundance data are available for the light $r$-process elements.

Since we are interested in determining where this second nucleosynthesis process, the LEPP, operates, we explore here the possibility of a neutron-capture process being responsible for the 
LEPP. We do not make any assumptions about the neutron exposure, but rather determine the necessary conditions to reproduce the newly obtained LEPP abundance pattern with site-independent full network calculations. Our goal is to constrain the neutron densities and temperatures needed for a LEPP process in order to be consistent with observations. In particular, we want to determine whether the LEPP is an $s$ - or an $r$-process (different from the one creating the $r$-II abundances).

We use a classical approach with a constant neutron exposure of neutron density $n_{n}$ and duration $\tau$ at a temperature $T$. We vary conditions from $s$-process-type conditions to $r$-process-type. We do not use any waiting point or steady flow approximation but employ a full reaction network for the abundances of 3224 nuclei from $\mathrm{H}$ to $\mathrm{Ta}$, taking into account neutron-capture rates, their inverse $(\gamma, n)$ photodisintegration rates, and $\beta$-decay rates with $\beta$-delayed neutron emission. The nuclear reaction rates were taken from the recent REACLIB compilation, which includes theoretical reaction rates based on NON-SMOKER statistical model calculations ( Rauscher \& Thielemann 2000) with $Q$-values obtained with the FRDM (Möller et al. 1995) mass model. Experimental Maxwellian average neutron-capture cross sections and their temperature trends were taken from Bao et al. (2000) when available. Experimental $\beta$-decay rates were used when available Pfeiffer et al. (2002). ${ }^{12}$ Theoretical $\beta$-decay rates were taken from Möller et al. (1997) or, when available, from calculations including first forbidden transitions (Möller et al. 2003). Temperatureand density-dependent $\beta$-decay rates from Takahashi \& Yokoi (1987) were included. The temperature was kept constant as a function of time. The initial abundance composition consisted of neutrons and seed nuclei, either ${ }^{56} \mathrm{Fe},{ }^{40} \mathrm{Ca}$, or a solar distribution seed. The ${ }^{56} \mathrm{Fe}$ mass fraction used in the calculation was $1 \%$. The neutron-to-seed ratio was chosen such that $n_{n}$ did not change by more than 5\% during the calculation. The choice of seed abundance does not affect the final abundance pattern, as long as it is below the mass region of interest, and therefore none of our conclusions depend on it. Our choice of Fe as seed is arbitrary and a purely technical means to create a neutron-capture flow through the relevant mass region. In particular, it does not imply that the process is of secondary nature. In general, a neutron-capture process requires some seed. In the case of a primary process, this seed had to be created in the same astrophysical event. An example is the $\alpha$-process generating the seed for the $r$-process in the neutrinodriven wind scenario in core-collapse supernovae.

To quantify which conditions better fit the stellar LEPP abundance pattern, a $\chi^{2}$ function $f\left(n_{n}, T, \tau\right)$, defined as

$$
f\left(n_{n}, T, \tau\right)=\sum_{i \in \mathrm{LEPP}}\left(\frac{Y_{i}^{\mathrm{CAL}}-Y_{i}^{\mathrm{LEPP}}}{\Delta Y_{i}}\right)^{2},
$$

was used, where $Y_{i}^{\mathrm{CAL}}$ is the calculated stable abundance and $Y_{i}^{\mathrm{LEPP}}$ is the desired abundance of element $i$. The closer the value of $f\left(n_{n}, T, \tau\right)$ is to the number of residuals, the better the agreement between the calculated abundance pattern and the stellar LEPP abundance pattern. Since the size of the uncertainty of the reference abundances for all elements is relatively the same, the $\chi^{2}$ function is not dominated by one uncertainty, and its use is justified.

The duration for the neutron exposure $\tau$ was chosen to minimize $f\left(n_{n}, T, \tau\right)$ for a given set of astrophysical conditions $n_{n}$ and $T$. In order to do this, we started the calculation for a given $T$ and $n_{n}$, and for every time step we determined the abundance pattern

\footnotetext{
12 National Nuclear Data Center, http://www.nndc.bnl.gov.
}

that would be produced if the neutrons were instantly exhausted at that point and all nuclei decayed back to stability via $\beta$-decays. The use of a full decay network including $\beta$-delayed neutron emission for this purpose at every time step and for all conditions was computationally impracticable. The $\beta$-delayed neutron emission was, however, included when calculating the final abundance pattern for the optimum process duration.

The resulting best abundance patterns for different conditions are shown in Figure 6. These calculations were performed with a ${ }^{56} \mathrm{Fe}$ seed. Using ${ }^{40} \mathrm{Ca}$ or a solar abundance distribution as seed did not have a major impact on the abundance pattern, but only changed the neutron flux duration $\tau$. We find that the abundances of elements $38 \leq Z \leq 47$ can be reasonably reproduced under a variety of different astrophysical conditions. Even though low neutron densities $n_{n} \approx 10^{8} \mathrm{~cm}^{-3}$ and high neutron densities $n_{n} \approx$ $10^{28} \mathrm{~cm}^{-3}$ can fit the LEPP pattern best in this region, other neutron densities can reproduce the pattern within a factor of 3 for every element. However, if heavier elements $(Z \geq 56)$, even in a relatively low amount such as in the LEPP, also have to be created, low neutron densities are favored to reproduce the desired abundances. This is illustrated in Figure 7, which displays $f\left(n_{n}, T, \tau\right)$ for different astrophysical conditions. Neutron-capture processes with a low neutron density, $n_{n} \leq 10^{13} \mathrm{~cm}^{-3}$, reproduce the residual abundance pattern better. Higher neutron densities fail to reproduce the abundance pattern, since it is not possible (within the model) to create the correct abundances for $38 \leq Z \leq 47$ and sufficient amounts of $Z \geq 56$ material. The dependence on temperature is small; only for low neutron densities can a high temperature be excluded.

The average atomic number of the created nuclei increases as a function of time as neutron captures are followed by $\beta$-decays. As the material becomes heavier, some of it reaches the region $38 \leq Z \leq 47$, and the desired abundance pattern may be reproduced. As more and more material increases its atomic number, the abundance in the region $38 \leq Z \leq 47$ decreases and the abundance of $Z \geq 56$ starts to increase. To satisfactorily reproduce the residual abundance pattern, most of the abundance has to go into $38 \leq Z \leq 47$. For $Z \geq 56$, the amount of created material has to be about 1 order of magnitude less than the average abundance of the light elements. The neutron shell closure $N=82$ is a bottleneck where abundances accumulate. In order to produce sufficient $38 \leq Z \leq 47$ abundances, the neutron flux has to be exhausted while most of the material is passing through the $N=82$ bottleneck. For processes with a large neutron density, the abundance peak occurs around $50 \leq Z \leq 56$. In order to produce elements $56 \leq Z \leq 62$, enough material has to leak out of the bottleneck. Because of the relatively long time for that to occur, the abundance of elements $Z=47$ and 48 already decreases before enough $56 \leq$ $Z \leq 62$ material is created. For processes with a relatively small neutron density, the shell closure produces progenitor bottleneck abundances in the region $56 \leq Z \leq 60$; therefore, the required amount of heavy material can still be obtained.

Even though low neutron density scenarios produce a higher amount of heavy material that is in agreement with the solar LEPP abundances, the stellar LEPP abundance pattern is not completely reproduced for $Z \geq 56$, as shown in Figure 6. While Ba seems to always be overproduced, $\mathrm{Pr}$ and $\mathrm{Sm}$ are underproduced by the network model using low neutron density scenarios. The neutron flux duration necessary to obtain a reasonable fit under the lowest neutron density $\left(\tau \approx 1170 \mathrm{yr}\right.$ for $n_{n}=10^{8} \mathrm{~cm}^{-3}$ ) far exceeds what is expected by present nucleosynthesis calculations in massive stars (Woosley et al. 2002; Rauscher et al. 2002; Chieffi \& Limongi 2004). More favorable conditions would require higher $n_{n}$. The choice $n_{n}=10^{13} \mathrm{~cm}^{-3}$ implies $\tau \approx 5$ days. A primary neutron 

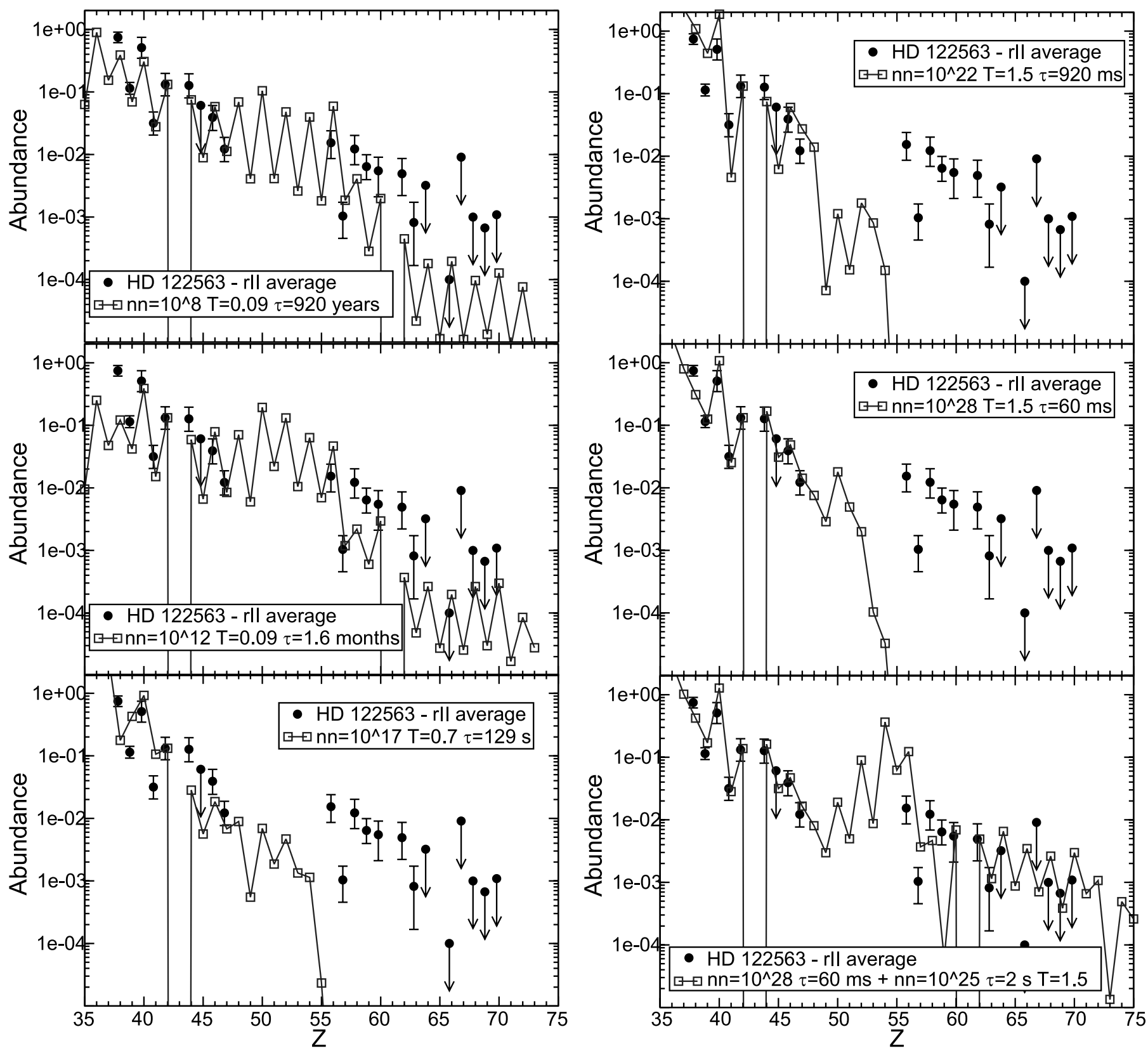

FIG. 6.-Abundances as a function of atomic number normalized to Mo for different astrophysical conditions and compared with the stellar LEPP (HD $122563-r$-II

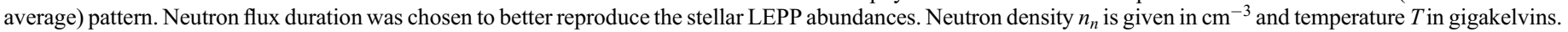
[See the electronic edition of the Journal for a color version of this figure.]

source would also be necessary to obtain a primary-LEPP mechanism, either by the elusive channel ${ }^{12} \mathrm{C}+{ }^{12} \mathrm{C} \rightarrow n+{ }^{23} \mathrm{Mg}$ or by more sophisticated situations in which convective shell C-burning layers merge with hotter inner regions suffering $\mathrm{Ne}$-shell and O-shell burning in the most advanced phases. These developments are outside the scope of the present analysis.

Even though in the high end of the $n_{n} \leq 10^{13} \mathrm{~cm}^{-3}$ range some amount of ${ }^{129} \mathrm{I}$ is made (at a neutron density $n_{n} \approx 10^{11} \mathrm{~cm}^{-3}$ the neutron capture on ${ }^{127} \mathrm{Te}$ becomes comparable to the $\beta$-decay halflife $\approx 10 \mathrm{hr}$, and therefore a subsequent neutron capture on the stable ${ }^{128} \mathrm{Te}$ and the ${ }^{129} \mathrm{Te} \beta$-decay creates it), in our calculations not enough ${ }^{127} \mathrm{I}$ is produced to explain the meteoritic ratios. However, it is interesting that for low neutron densities, $n_{n} \leq 10^{11} \mathrm{~cm}^{-3}$, the derived solar LEPP isotopic abundances are in agreement with the missing $s$-process abundances in the region Mo-Xe predicted by the Galactic chemical evolution model by Travaglio et al. (2004) that includes the yields of all AGB stars according to their lifetimes and production at various metallicities. In particular, $s$-only isotopes in the region from Mo to Xe are within $20 \%-$ $30 \%$ of their solar abundances. For higher neutron densities, the LEPP isotopic distribution shifts progressively toward an $r$-process behavior.

The disadvantage of using a site-independent model is that the reaction network calculations may be overly simplistic and some important features can be left out. Such $r$-process scenarios as the neutrino-driven wind in supernovae and supernova fallback (Woosley \& Hoffman 1992; Takahashi et al. 1994; Wanajo et al. 2001; Thompson et al. 2001; K. Farouqi et al. 2008, in preparation; Fryer et al. 2006) have neutron densities that dramatically evolve with time. By keeping a single constant neutron density the effect of such change cannot be correctly reproduced. For such a reason we also performed test calculations to explore whether 


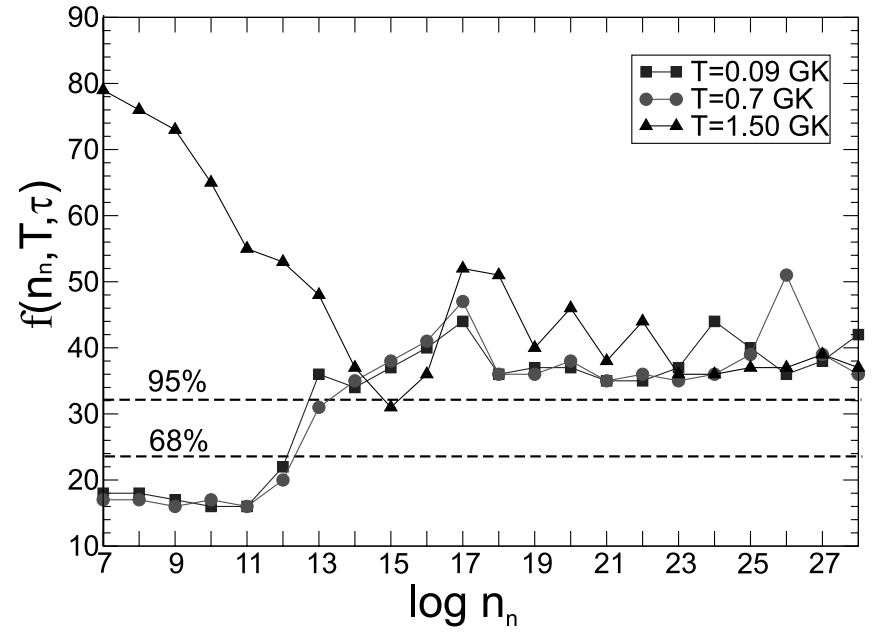

FIG. 7.-The $f\left(n_{n}, T, \tau\right)$ as function of neutron density $n_{n}$ for different temperatures when comparing the results of the network calculations with the modified HD 122563 abundance pattern. Confidence intervals for the $\chi^{2}$ distribution are also shown. [See the electronic edition of the Journal for a color version of this figure.]

separate $r$-process components for the $38 \leq Z \leq 47$ and $56 \leq$ $Z \leq 62$ regions could reproduce the LEPP abundance pattern. Figure 6 shows the abundance pattern when using components $n_{n}=10^{28} \mathrm{~cm}^{-3}, \tau=60 \mathrm{~ms}$ and $n_{n}=10^{25} \mathrm{~cm}^{-3}, \tau=2 \mathrm{~s}$ at $T_{9}=1.5$. Although the stellar LEPP is not completely reproduced for the heavy elements, the abundance pattern is reproduced to better than an order of magnitude, with the exception of $\mathrm{Ba}$ and Pr. Even though the choice of components is not unique, a lower neutron density limit of $n_{n}=10^{24} \mathrm{~cm}^{-3}$ was found preferable to reproduce light and heavy elements without overproducing $\mathrm{Pd}$ and $\mathrm{Ag}$, although some overproduction of Ba cannot be avoided. In this case, no $s$-process production is possible. Site-dependent calculations should be performed in the future to compare the observed LEPP abundances with predictions from various realistic scenarios.

\section{CONCLUSIONS}

We have shown that the elemental abundances of metal-poor halo stars exhibit a noncorrelation between $[\mathrm{X} / \mathrm{Eu}]$ and $[\mathrm{Eu} / \mathrm{Fe}]$ for Y, Sr, Zr, Pd, and Ag. The same behavior had been found before for $\mathrm{Y}$ and $\mathrm{Zr}$ in a few stars in M15 (Otsuki et al. 2006). This provides further evidence for the existence of a primary LEPP process that contributes, together with the $r$-process, the weak $s$-process, the main $s$-process, and the $p$-process, to the nucleosynthesis of not only $\mathrm{Y}, \mathrm{Sr}$, and $\mathrm{Zr}$ but, as we show here, most elements in the $\mathrm{Sr}-\mathrm{Ag}$ range. We also find that a very small contribution to still heavier elements up to Eu is likely. Based on our results we were then able to show that the LEPP produces a uniform and unique abundance pattern, shown in Figure 5, and together with the pattern observed in Eu-enriched stars ( $r$-process rich) is able to explain the abundances of all metal-poor stars considered.

Metal-poor stars with very weak Eu enhancement play an essential role in constraining the LEPP, as they have the smallest contribution from the $r$-process. A prime example is HD 122563 , for which a wide range of elemental abundances are observed. We are therefore able to explain the abundance observations in HD 122563 and HD 221170 that previously had been identified as "anomalies," together with the abundances observed in other metal-poor halo stars, with a consistent picture of mixed contributions from the $r$-process and the LEPP. In addition, it was found that the LEPP contributes significantly to the solar system abundances based on the use of the Travaglio et al. (2004) $s$-process model. While we consider this model to be the best available, it should be noted that the use of the simpler $s$-process model by Arlandini et al. (1999), for example, would have led to a significantly reduced solar system contribution of the LEPP. However, only when using the Travaglio et al. (2004) $s$-process model do the solar and stellar LEPP abundance patterns agree.

Since the astrophysical conditions that would create the LEPP abundance pattern are not known, full reaction network calculations were performed in a heuristic way assuming different neutroncapture-process conditions. A variety of different neutron densities from $s$-process-like to $r$-process-like were found to reproduce the abundance pattern between $\mathrm{Sr}$ and $\mathrm{Ag}$ within the observational uncertainties. However, intermediate neutron densities in between typical $s$ - or $r$-process conditions seem to be excluded. When using a single component to reproduce the LEPP pattern, only neutron densities $n_{n} \leq 10^{13} \mathrm{~cm}^{-3}$ seem to create enough Ba to Sm material (which actually consists of quite small contributions to solar) that is consistent within an order of magnitude with the abundances inferred for HD 122563. These low neutron densities correspond to densities found in the $s$-process or not too far from it. A LEPP characterized by neutron densities of $n_{n} \leq 10^{13} \mathrm{~cm}^{-3}$ then addresses not only the problem of explaining metal-poor star abundance patterns but also the problem of the underproduction of some $s$-only isotopes in the $s$-process Galactic chemical evolution model of Travaglio et al. (2004).

Multiple nucleosynthesis processes are also required to explain the early solar system ${ }^{129} \mathrm{I} /{ }^{127} \mathrm{I}$ and ${ }^{182} \mathrm{Hf} /{ }^{180} \mathrm{Hf}$ ratios inferred from meteorites (Qian \& Wasserburg 2003). As ${ }^{129} \mathrm{I}$ and ${ }^{182} \mathrm{Hf}$ are radioactive nuclei with different half-lives, the detected abundance ratios imply different chemical evolution histories for ${ }^{129} \mathrm{I}$ and ${ }^{182} \mathrm{Hf}$, both thought to be produced in the $r$-process. If low neutron density scenarios are responsible for the LEPP, the $A=130$ abundance peak could be attributed to the main $r$-process component (as observed in $r$-II metal-poor stars). In this case, most of ${ }^{129} \mathrm{I}$ and ${ }^{182} \mathrm{Hf}$ would be produced in the same $r$-process events, which could not explain the meteoritic data. It should be noted, however, that Meyer \& Clayton (2000) have questioned the pure $r$-process origin of ${ }^{182} \mathrm{Hf}$. An alternative scenario that would satisfy the meteoritic constraints would be that yet another process is responsible for the origin of the $A=130$ abundance peak. This would require the $A=130$ production, or at least the production of ${ }^{129} \mathrm{I}$, to be largely avoided in the main $r$-process that is known to produce the heavy elements from $\mathrm{Ba}$ and beyond. Similarly, the hypothetical additional process that is responsible for the synthesis of ${ }^{129}$ I would have to provide negligible contributions to $\mathrm{Ba}$. It would have to be demonstrated in realistic model calculations that both requirements can be achieved. Recent studies based on the classical $r$-process model indicate that this might be difficult given the known nuclear physics around the $N=82$ shell closure (Kratz et al. 2007).

The astrophysical scenarios involving neutron densities $n_{n} \leq$ $10^{13} \mathrm{~cm}^{-3}$ do not correspond to the traditional weak or main $s$-process, because the nucleosynthesis occurs in very low metallicity stars and the required neutron flux duration is too long compared to what is expected in those scenarios. A particular challenge is to find a stellar scenario with low neutron densities during a long period of time occurring in low-metallicity stars strong enough to produce elements up to $\mathrm{Eu}$. Since it is hard to envision such a scenario, possibilities other than low neutron-capture processes should also be considered to explain the observed LEPP abundances.

While it is not possible to reproduce the entire LEPP abundance pattern at high neutron densities with a single neutron exposure, 
we showed that, in principle, a multicomponent exposure with neutron densities $n_{n} \geq 10^{24} \mathrm{~cm}^{-3}$ could reproduce the observed abundances. In such a model the LEPP could synthesize ${ }^{129} \mathrm{I}$ explaining the meteoritic data, although the overproduction of $\mathrm{Ba}$ is difficult to avoid. A high neutron density LEPP would, however, require that the solar abundance residual (our solar LEPP abundance pattern) cannot, or at least not entirely, be explained with LEPP anymore, as it contains the $s$-only nuclei underproduced in Travaglio et al. (2004). In this case one would have to conclude that the LEPP contributes at most a small amount to the solar abundances, and that an unknown additional $s$-process component is required to explain the solar abundances. Moreover, the agreement between our solar LEPP pattern and the observed LEPP component in metal-poor stars pointed out in this work would then have to be considered coincidental.

The $s$-process contribution to solar ${ }^{96} \mathrm{Mo}$ is only $78 \%$. Since that isotope is shielded by ${ }^{96} \mathrm{Ru}$ against an $r p$-process far from stability, one might argue that such a process is possibly excluded as an explanation for the LEPP. Nevertheless, a nucleosynthesis process on the proton-rich side running closer to stability, such as the recently proposed $\nu p$-process, should be considered. Besides proton captures, the $\nu p$-process includes neutron-induced reactions and therefore has a path closer to stability producing isotopes such as ${ }^{96} \mathrm{Mo}$. In addition, Wanajo (2006) has shown that the $\nu$-process under some conditions can produce enough material up to Eu. Further studies should also consider this process a candidate for the production of LEPP abundances.

The parameter study in the present work is a first step toward determining the astronomical site responsible for creating the abundance of material not created in the $r$-process in metal-poor stars. More observational data, particularly for $r$-process-poor stars and more elements below Ba, would certainly be important for further progress. It would also be desirable to identify actual sites that could be responsible for the LEPP and perform sitespecific calculations to reproduce our derived LEPP abundance pattern.

After submission of this paper, Qian \& Wasserburg (2007) presented a refinement of their phenomenological model that is based on observational constraints similar to those presented here. Their model is based on the observed abundances in HD 122563 (for their "L" component) and CS 22892-052 (for their " $H$ " component). Although their adopted patterns are slightly different, their conclusion that such a two-component model can explain currently available metal-poor star abundance patterns is in agreement with this work. Our results concerning the implications of the production of some $A \geq 130$ nuclei in the LEPP, the likely nature of the LEPP, and its potential relevance for the $s$-process are not affected.

We thank I. I. Ivans, J. E. Lawler, C. Sneden, and K. Lodders for helpful discussions and F.-K. Thielemann for providing the reaction network solver. This work has been supported in part by NSF grants PHY 02-16783 (Joint Institute for Nuclear Astrophysics) and PHY 01-10253. Support was also provided by NSF grants AST 03-07279 (J. J. C.) and AST 04-06784 (T. C. B.), by the Virtuelles Institut für Struktur der Kerne und Nukleare Astrophysik under HGF grant VH-VI-061, by the Univ. Mainz-GSI F+E Vertrag under grant MZ/KLK (K.-L. K., B. P., and K. F.), and by the Italian MIUR-FIRB Project "Astrophysical Origin of the Heavy Elements Beyond Fe" (R. G.).
Anders, E., \& Grevesse, N. 1989, Geochim. Cosmochim. Acta, 53, 197

Aoki, W., et al. 2005, ApJ, 632, 611

Arlandini, C., Käppeler, F., Wisshak, K., Gallino, R., Lugaro, M., Busso, M., \& Straniero, O. 1999, ApJ, 525, 886

Bao, Z. Y., Beer, H., Käeppeler, F., Voss, F., Wisshak, K., \& Rauscher, T. 2000, At. Data Nucl. Data Tables, 76, 70

Barklem, P. S., et al. 2005, A\&A, 439, 129

Beers, T. C., \& Christlieb, N. 2005, ARA\&A, 43, 531

Burris, D. L., Pilachowski, C. A., Armandroff, T. A., Sneden, C., Cowan, J. J., \& Roe, H. 2000, ApJ, 544, 302

Cameron, A. G. W. 2001, ApJ, 562, 456

Chieffi, A., \& Limongi, M. 2004, ApJ, 608, 405

Christlieb, N., et al. 2004, A\&A, 428, 1027

Clayton, D. D., \& Rassbach, M. E. 1967, ApJ, 148, 69

Cowan, J. J., Sneden, C., Lawler, J. E., \& Den Hartog, E. A. 2006, in Nuclei in the Cosmos IX, ed. J. Cederkall et al. (Trieste: SISSA), 14

Cowan, J. J., Thielemann, F.-K., \& Truran, J. W. 1991, Phys. Rep., 208, 267

Cowan, J. J., et al. 2002, ApJ, 572, 861

Cristallo, S., Straniero, O., Gallino, R., Piersanti, L., \& Dominguez, I. 2006, in Nuclei in the Cosmos IX, ed. J. Cederkall et al. (Trieste: SISSA), 62

Freiburghaus, C., Rosswog, S., \& Thielemann, F.-K. 1999, ApJ, 525, L121

Fröhlich, C., Martinez-Pinedo, G., Liebendörfer, M., Thielemann, F.-K., Bravo, E., Hix, W. R., Langanke, K., \& Zinner, N. T. 2006, Phys. Rev. Lett., 96, 142502

Fryer, C. L., Herwig, F., Hungerford, A., \& Timmes, F. X. 2006, ApJ, 646, L131

Goriely, S., \& Arnould, M. 2001, A\&A, 379, 1113

Goriely, S., Demetriou, P., Janka, H.-Th., Pearson, J. M., \& Samyn, M. 2005, Nucl. Phys. A, 758, 587

Hill, V., et al. 2002, A\&A, 387, 560

Honda, S., Aoki, W., Ishimaru, Y., \& Wanajo, S. 2007, ApJ, 666, 1189

Honda, S., Aoki, W., Ishimaru, Y., Wanajo, S., \& Ryan, S. G. 2006, ApJ, 643, 1180

Honda, S., Aoki, W., Kajino, T., Ando, H., Beers, T. C., Izumiura, H., Sadakena, K., \& Takada-Hidai, M. 2004, ApJ, 607, 474

Ishimaru, Y., Wanajo, S., Aoki, W., Ryan, S. G., \& Prantzos, N. 2005, Nucl. Phys. A, 758,603

Ivans, I. I., Simmerer, J., Sneden, C., Lawler, J. E., Cowan, J. J., Gallino, R., \& Bisterzo, S. 2006, ApJ, 645, 613

\section{EFERENCES}

Johnson, J. A., \& Bolte, M. 2002, ApJ, 579, 616

Käppeler, F., Beer, H., Wisshak, K., Clayton, D. D., Macklin, R. L., \& Ward, R. A. 1982, ApJ, 257, 821

Kratz, K.-L., Bitouzet, J.-P., Thielemann, F.-K., Moeller, P., \& Pfeiffer, B. 1993 , ApJ, 403, 216

Kratz, K.-L., Farouqi, K., Pfeiffer, B., Truran, J. W., Sneden, C., \& Cowan, J. J. 2007, ApJ, 662, 39

Lambert, D. L., \& Allende Prieto, C. 2002, MNRAS, 335, 325

Lodders, K. 2003, ApJ, 591, 1220

McWilliam, A. 1998, AJ, 115, 1640

Meyer, B. S., \& Clayton, D. D. 2000, Space Sci. Rev., 92, 133

Möller, P., Nix, J. R., \& Kratz, K.-L. 1997, At. Data Nucl. Data Tables, 66, 131

Möller, P., Nix, J. R., Myers, W. D., \& Swiatecki, W. J. 1995, At. Data Nucl.

Data Tables, 59, 185

Möller, P., Pfeiffer, B., \& Kratz, K.-L. 2003, Phys. Rev. C, 67, 055802

Norris, J. E., Ryan, S. G., \& Beers, T. C. 2001, ApJ, 561, 1034

Otsuki, K., Honda, S., Aoki, W., Kajino, T., \& Mathews, G. J. 2006, ApJ, 641, L117

Pfeiffer, B., Kratz, K.-L., \& Möller, P. 2002, Prog. Nucl. Energy, 41, 39

Pfeiffer, B., Kratz, K.-L., Thielemann, F.-K., \& Walters, W. B. 2001a, Nucl.

Phys. A, 693, 282

Pfeiffer, B., Ott, U., \& Kratz, K.-L. 2001b, Nucl. Phys. A, 688, 575

Pignatari, M., et al. 2006, in Nuclei in the Cosmos IX, ed. J. Cederkall et al. (Trieste: SISSA), 61

Qian, Y. Z., Vogel, P., \& Wasserburg, G. J. 1998, ApJ, 494, 285

Qian, Y. Z., \& Wasserburg, G. J. 2000, Phys. Rep., 333, 77 2001, ApJ, 559, 925

2003, ApJ, 588, 1099

2007, Phys. Rep., 442, 237

Raiteri, C. M., Gallino, R., \& Busso, M. 1992, ApJ, 387, 263

Rauscher, T., Heger, A., Hoffman, R. D., \& Woosley, S. E. 2002, ApJ, 576, 323 Rauscher, T., \& Thielemann, F.-K. 2000, At. Data Nucl. Data Tables, 75, 1

Rosswog, S., Liebendörfer, M., Thielemann, F.-K., Davies, M. B., Benz, W., \& Piran, T. 1999, A\&A, 341, 499

Schatz, H., Toenjes, R., Pfeiffer, B., Beers, T. C., Cowan, J. J., Hill, V., \& Kratz,

K.-L. 2002, ApJ, 579, 626

Sneden, C., et al. 2003, ApJ, 591, 936 
Surman, R., \& McLaughlin, G. C. 2005, Nucl. Phys. A, 758, 189

Takahashi, K., Witti, J., \& Janka, H.-T. 1994, A\&A, 286, 857

Takahashi, K., \& Yokoi, K. 1987, At. Data Nucl. Data Tables, 36, 375

Thompson, T. A., Burrows, A., \& Meyer, B. S. 2001, ApJ, 562, 887

Travaglio, C., Gallino, R., Arnone, E., Cowan, J. J., Jordan, F., \& Sneden, C. 2004, ApJ, 601, 864

Travaglio, C., Gallino, R., Busso, M., \& Gratton, R. 2001, ApJ, 549, 346

Truran, J. W., Cowan, J. J., Pilachowski, C. A., \& Sneden, C. 2002, PASP, 114, 1293
Vockenhuber, C., et al. 2004, Phys. Rev. Lett., 93, 172501

Wanajo, S. 2006, ApJ, 647, 1323

Wanajo, S., Kajino, T., Mathews, G. J., \& Otsuki, K. 2001, ApJ, 554, 578

Wasserburg, G. J., Busso, M., \& Gallino, R. 1996, ApJ, 466, L109

Wasserburg, G. J., \& Qian, Y.-Z. 2000a, ApJ, 529, L21 . 2000b, ApJ, 538, L99

Westin, J., Sneden, C., Gustafsson, B., \& Cowan, J. J. 2000, ApJ, 530, 783

Woosley, S. E., Heger, A., \& Weaver, T. A. 2002, Rev. Mod. Phys., 74, 1015 Woosley, S. E., \& Hoffman, R. D. 1992, ApJ, 395, 202 